\title{
A Case of Multi-System Langerhans Cell Histiocytosis with Local Invasion of the Orbital Apex
}

\author{
Hiroko Yamada ${ }^{a}$ Takuji Kurimoto ${ }^{a}$ Sotaro Mori ${ }^{a}$ Mari Sakamoto ${ }^{a}$ \\ Kaori Ueda ${ }^{a}$ Yuko Yamada-Nakanishi ${ }^{a}$ Yoichi Uozumi ${ }^{b}$ \\ Hiroyasu Shose ${ }^{b}$ Masaaki Taniguchi ${ }^{b}$ Masanori Toyoda ${ }^{c}$ \\ Hironobu Minamic Makoto Nakamura ${ }^{a}$ \\ aDivision of Ophthalmology, Department of Surgery, Kobe University Graduate School of \\ Medicine, Kobe, Japan; 'bepartment of Neurosurgery, Kobe University Graduate School of \\ Medicine, Kobe, Japan; 'Division of Medical Oncology/Haematology, Department of \\ Medicine, Kobe University Graduate School of Medicine, Kobe, Japan
}

\section{Keywords}

Langerhans cell histiocytosis · Optic nerve · Orbital apex

\begin{abstract}
Langerhans cell histiocytosis $(\mathrm{LCH})$ is characterised by tissue destruction caused by the abnormal proliferation of pathogenic dendritic cells. We report a rare case of multi-system LCH with local invasion of the orbital apex. A 56-year-old woman suffered from a decrease of visual acuity in the left eye caused by central scotoma and the limitation of eye movement in all directions. Magnetic resonance imaging revealed an enhanced lesion in the left orbital apex, suggesting optic nerve compression. She had been diagnosed with eosinophilic granuloma 24 years previously. Two weeks after the current presentation, we admitted the patient for optic canal and orbital apex decompression and subtotal tumour resection. Histopathological analysis confirmed the diagnosis of LCH. Post-surgical treatment with low-dose cytarabine was initiated for the residual tumour. However, it was ceased because of myelosuppression-induced pyelonephritis. After surgery, the central scotoma disappeared on day 5 and eye movement palsy resolved by 6 months. After the cessation of cytarabine, she has received low-dose
\end{abstract}


steroid therapy for 2 years with no recurrence. Early surgical intervention with low-dose steroid therapy can lead to recovery of visual acuity and resolve eye movement palsy in patients with lesions of the orbital apex caused by multi-system LCH.

\section{Introduction}

Langerhans cell histiocytosis (LCH) causes proliferation of pathogenic dendritic cells in various organs such as the skin, bones, liver, and pituitary gland that results in tissue damage and destruction. However, involvement of the visual pathway is infrequent [1-5]. When parts such as the optic nerve or optic chiasm are involved, some reports suggest that surgical treatment can improve visual acuity $[4,5]$. Here, we report a rare case of multi-system LCH presenting acutely with ophthalmic symptoms due to local invasion of the orbital apex. Early surgical intervention led to the complete recovery of visual acuity and eye movement with lowdose oral prednisolone.

\section{Case Presentation}

A 56-year-old Japanese woman suddenly experienced diplopia while driving a car and was diagnosed as having abducens nerve palsy. Head magnetic resonance image (MRI) showed no abnormalities at that time. One month later, the best-corrected visual acuity (BCVA) of her left eye decreased to 0.3 and her left eye developed a relative afferent pupillary defect. She was then referred to our clinic for further investigation. She had been diagnosed with eosinophilic granuloma 24 years previously. Diagnosis at that time was based on the histopathological results and multiple osteolytic lesions being present in the occipital and temporal bones. She then received surgery, steroid therapy, and chemotherapy with etoposide and vinblastine; however, therapy was terminated because of myelosuppression as a complication of the chemotherapy.

At our initial examination, the patient's BCVAs were 1.0 and 0.4 in the right and left eyes, respectively, with corresponding intraocular pressures of 18 and $17 \mathrm{~mm} \mathrm{Hg}$. The light reflex of the left eye was sluggish and incomplete, with a relative afferent pupillary defect, and the critical fusion frequency of the left eye was only $19 \mathrm{~Hz}$. In addition, the alternative prism cover test showed esotropia of 2 prism diopters and left/right hypertropia of 10 prism diopters at near distance as well as esotropia of 8 prism diopters and left-right hypertropia of 16 prism diopters at far distance. The 9-gaze view showed almost normal eye movement in all directions, but a binocular single vision test showed diplopia in the entire visual field. There was no anisocoria and she had a normal corneal reflex.

Further investigation by Goldmann perimetry revealed a central scotoma of the left eye (Fig. 1a), but no abnormalities in the right eye (Fig. 1b). MRI revealed invasive lesions with homogeneous enhancement by gadolinium adjacent to the lesser wing of the sphenoid bone that appeared to extend toward the ipsilateral optic canal. We presumed that the lesion was compressing the optic nerve where it had expanded from the optic canal to the orbital apex, with optic nerve compression developing from a superolateral to an inferomedial direction (Fig. 2a-c).

Two weeks after the initial examination, we admitted the patient for optic canal and orbital apex decompression and subtotal tumour resection via a transcranial approach. In particular, we focused on sufficiently opening the optic canal. We chose this approach based on 


\section{Case Reports in Ophthalmology}

the MRI findings of invasion around the optic nerve sheath, which suggested that there was significant compression of the optic nerve. Intra-operative findings revealed that inflammatory granulomatous tissue was compressing, but not tightly adherent to the optic nerve. Aiming to maximise the optic nerve decompression, we removed as much granulomatous tissue as possible from the sphenoid bone to the orbit. Rapid intra-operative histological diagnosis confirmed the presence of inflammatory cells and fibrous tissues, but no malignant tumour cells. Histopathology then showed a fibrous granuloma with marked inflammatory cell invasion and a fragmented osteogenic response. Dysplastic cells with a narrow shape in the middle and a swollen nucleus, resembling histiocytes, were also identified in the region with marked eosinophil infiltration. Finally, immunohistochemical analysis revealed that these dysplastic cells were positive for CD68, CD1a, and S100 antigens, allowing for a definitive diagnosis of LCH (Fig. 3).

From the day after surgery, the patient intravenously received hydrocortisone sodium phosphate at $100 \mathrm{mg} /$ day for 3 days and chemotherapy with low-dose cytarabine was initiated as a post-surgical treatment for the residual tumour. Two months later, chemotherapy was stopped because of pyelonephritis that was complicated by myelosuppression. Low-dose steroid therapy was continued after stopping chemotherapy. All changes in the left eye subsequently resolved after surgery: the central scotoma disappeared by 5 days, BCVA improved to 0.7 by 2 weeks, and the eye movement palsy completely disappeared and BCVA improved to 1.2 by 6 months (Fig. 1c). At her 2-year follow-up appointment, the patient has had no recurrence with a low maintenance dose of prednisolone (5 mg) (Fig. 2d).

\section{Discussion}

LCH has both inflammatory and tumorous characteristics, with some researchers advocating the disease concept "inflammatory myeloid tumour." Historically, LCH comprised 3 diseases: eosinophilic granulomas, Hand-Schuller-Christian disease, and Letter-Siwe disease. Currently, it is categorised as either single-system LCH (SS-LCH) or multi-system LCH (MSLCH). SS-LCH is further divided into that with unifocal or multifocal lesions, whereas MS-LCH involves multiple organs or systems, with or without the involvement of risk organs (i.e., haematopoietic, splenic, liver, and lungs) [6].

The therapeutic strategy of LCH differs by the clinical categorisation. For SS-LCH with a unifocal lesion, simple observation is usually possible because spontaneous remission is typical. When bone lesions are present and risk causing damage by compression, surgical resection can be attempted if it is considered possible [7, 8]. However, in some cases, lesions are hard to dissect or pose a threat to the central nervous system, such as those of the craniofacial bone. Even if these cases only present as a single lesion, chemotherapy should be performed in combination with minimum bone resection [7]. By contrast, for MS-LCH, chemotherapy with vinblastine and prednisolone is the standard first-line therapy, with cytarabine, doxorubicin, and ifosfamide applied in refractory cases. Our patient had past histories of diabetes insipidus and thyroid dysfunction, suggesting a diagnosis of MS-LCH. Although it would have been reasonable to opt for chemotherapy as the first-line treatment option, the patient presented with a rapid deterioration in visual acuity. Therefore, we considered that decompression surgery plus chemotherapy would be more appropriate to ensure visual recovery without recurrence.

There have been several reports of optic neuropathy induced by LCH [1-5]. Including the present case, we identified 7 cases of compressive optic neuropathy treated surgically by 
either a transcranial approach (5 cases) or an endoscopic nasal approach ( 2 cases). Two cases underwent craniotomy and subtotal resection of the tumour, but there was no description of the visual outcomes, and the other 3 cases reported improved visual functions. In both cases treated via the endoscopic nasal approach, visual function reportedly improved.

There are important differences between the transcranial and the endoscopic nasal approach. In the transnasal approach, lesions can be manipulated within approximately $120^{\circ}$ of the medial side of the optic canal, whereas in the transcranial approach, lesions can be manipulated within approximately $270^{\circ}$ of the outer side of the optic canal. Thus, the transnasal approach has a narrower operative field and is more restricted than the transcranial approach, but benefits from being less invasive. In the present case, MRI showed that there was an inflamed lesion in the orbital apex that was presumed to be compressing the optic nerve. Given the apparent extent, severity, and origin of the lesion, we chose the transcranial approach to remove as much of the continuous lesion as possible. Although chemotherapy failed because of a serious infectious complication, the patient has retained good visual function with only low-dose prednisolone therapy.

In a multicentre retrospective study, 14 of 1,665 patients $(0.84 \%)$ who underwent orbital surgery experienced severe vision loss that ranged from finger counting to no light perception [9]. However, the risk of blinding was shown to be increased significantly for the subset of patients undergoing surgery for orbital floor fracture repair in the setting of multiple facial fractures (6.45\%), bone decompression of the optic canal (15.6\%), or an intracranial approach to the orbital roof. Severe visual dysfunction developed in 5 of 32 cases that underwent decompression surgery of the optic canal. Of these, 4 and 1 cases underwent surgery by transcranial and transnasal approaches, respectively. Although decompression surgery of the optic canal carries a low risk of permanent damage, urgent decompression surgery after careful consideration of the risks and benefits can prove essential to the optimal recovery of visual function.

In conclusion, although it is rare, we should be aware of infiltration to the orbital apex when examining patients with LCH who present with visual dysfunction. Early surgical intervention may facilitate both accurate diagnosis and optimal visual outcomes.

\section{Acknowledgements}

We are grateful to Enago (www.enago.jp) for the English language review.

\section{Statement of Ethics}

This report was published with the permission and informed consent of the patient.

\section{Disclosure Statement}

None of the authors has any conflicts of interest. 


\section{Author Contributions}

Hiroko Yamada and Sotaro Mori acquired the data. Hiroko Yamada, Mari Sakamoto, and Kaori Ueda interpreted the data, and Hiroko Yamada wrote the draft of the manuscript. Takuji Kurimoto designed and conducted this study and revised the manuscript with support from Yuko Yamada-Nakanishi, Yoichi Uozumi, Hiroyasu Shose, Masaaki Taniguchi, Masanori Toyoda, Hironobu Minami, and Makoto Nakamura. All authors provided input to the drafting and critical revising of the manuscript. All authors read and approved the final version of the manuscript.

\section{References}

1 Belen D, Colak A, Ozcan OE. CNS involvement of Langerhans cell histiocytosis. Report of 23 surgically treated cases. Neurosurg Rev. 1996;19(4):247-52.

2 Gangwani V, Walker M, El-Defrawy H, Nicoll JA, Reck A, Pathmanathan T. Langerhans cell histiocytosis of the optic chiasm. Clin Exp Ophthalmol. 2007 Jan-Feb;35(1):66-8.

3 Hervey-Jumper SL, Ghori A, Ziewacz JE, McKeever PE, Chandler WF. Langerhans cell histiocytosis of the optic chiasm: case report. Neurosurgery. 2011 Feb;68(2):E556-61.

4 Bae JW, Kim YH, Kim SK, Wang KC, Shin HY, Kang HJ, et al. Langerhans cell histiocytosis causing acute optic neuropathy. Childs Nerv Syst. 2015 Apr;31(4):615-9.

5 Stromberg JS, Wang AM, Huang TE, Vicini FA, Nowak PA. Langerhans cell histiocytosis involving the sphenoid sinus and superior orbital fissure. AJNR Am J Neuroradiol. 1995 Apr;16(4 Suppl):964-7.

6 Haupt R, Minkov M, Astigarraga I, Schäfer E, Nanduri V, Jubran R, et al.; Euro Histio Network. Langerhans cell histiocytosis (LCH): guidelines for diagnosis, clinical work-up, and treatment for patients till the age of 18 years. Pediatr Blood Cancer. 2013 Feb;60(2):175-84.

7 Morimoto A, Ishida Y, Suzuki N, Ohga S, Shioda Y, Okimoto Y, et al.; HLH/LCH Committee of the Japanese Society of Pediatric Hematology. Nationwide survey of single-system single site Langerhans cell histiocytosis in Japan. Pediatr Blood Cancer. 2010 Jan;54(1):98-102.

8 Titgemeyer C, Grois N, Minkov M, Flucher-Wolfram B, Gatterer-Menz I, Gadner H. Pattern and course of single-system disease in Langerhans cell histiocytosis data from the DAL-HX 83- and 90-study. Med Pediatr Oncol. 2001 Aug;37(2):108-14.

9 Jacobs SM, McInnis CP, Kapeles M, Chang SH. Incidence, risk factors, and management of blindness after orbital surgery. Ophthalmology. 2018 Jul;125(7):1100-8. 
a
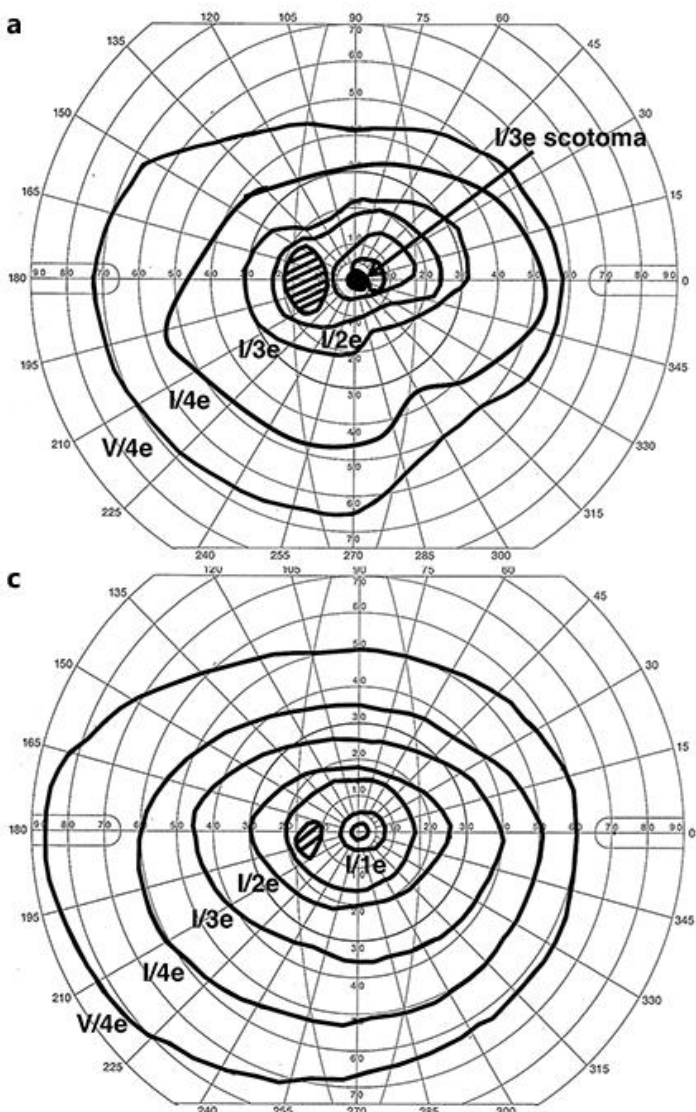

b

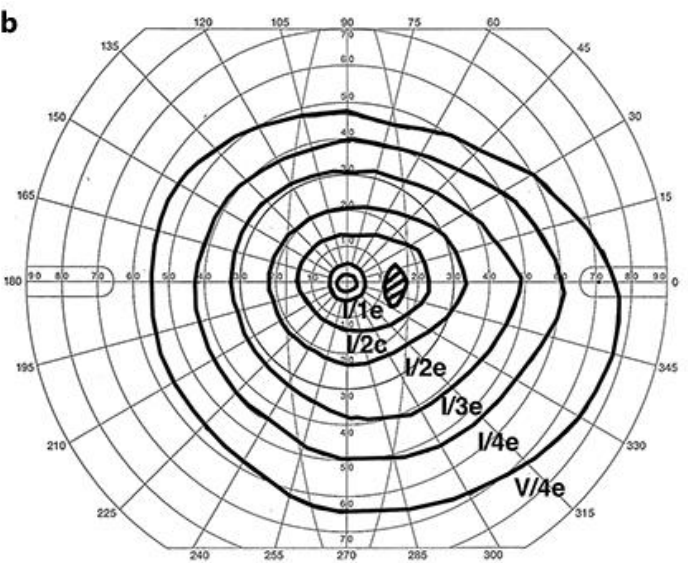

Fig. 1. Goldmann kinetic perimetry. Visual field results: before surgery in the left eye (a), before surgery in the right eye (b), and 5 days after surgery in the left eye (c). The small central scotoma of the left eye disappeared after surgery. 


\section{Case Reports in Ophthalmology}
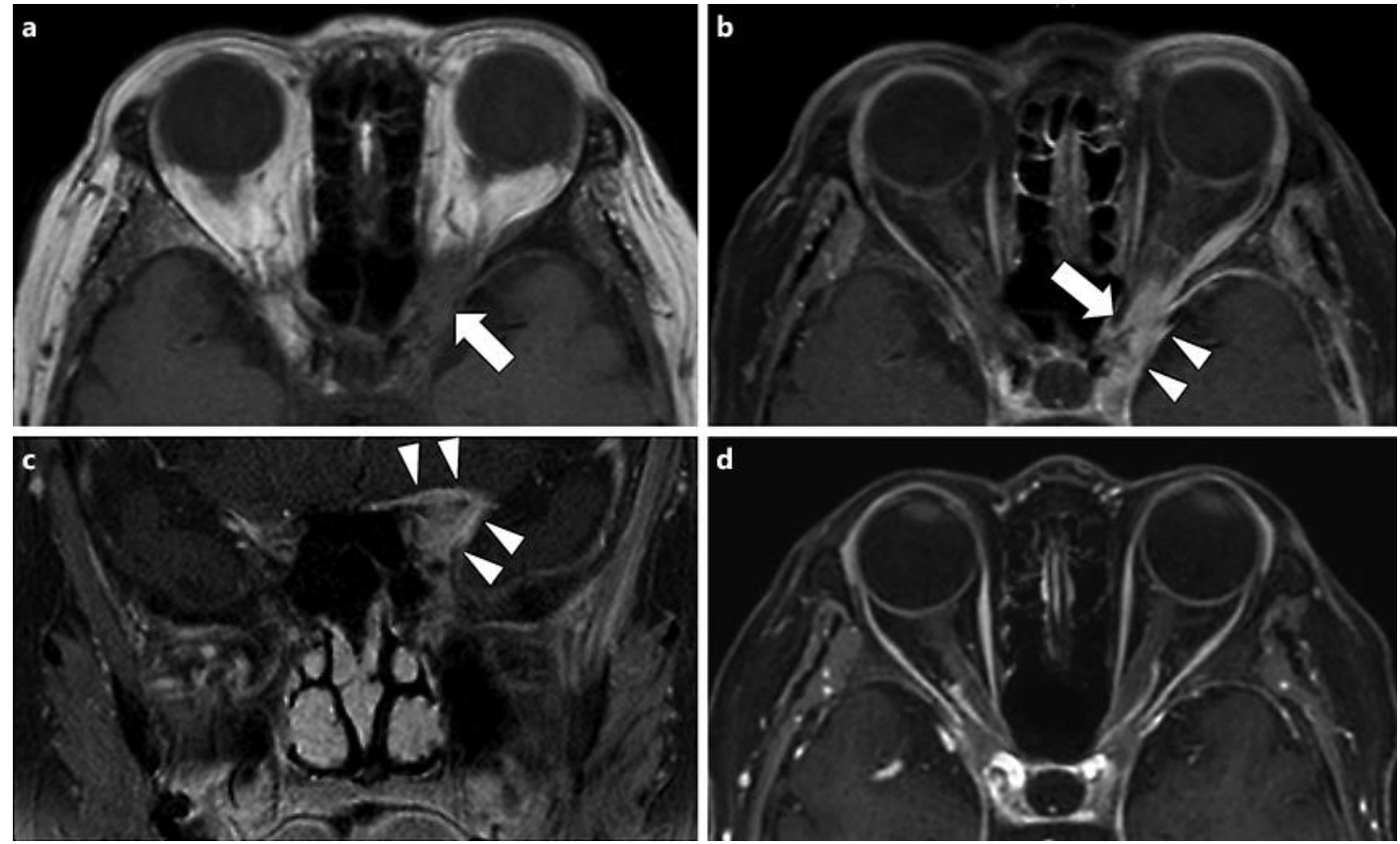

Fig. 2. Findings of orbital magnetic resonance image. The diagnostic T1-weighted images with gadolinium enhancement are shown in three views: axial (a), fat-suppressed axial (b), and coronal (c). The tumour was iso-intense in the T1-weighted image (arrow in a), was homogenously enhanced by gadolinium (arrow in b), and densely infiltrated the orbital apex, probably developing from the sphenoid bone (arrowheads in $\mathbf{b}$ and $\mathbf{c}$ ). $\mathbf{d}$ There was no tumour recurrence in the orbital apex on a fat-suppressed T1-weighted axial image with gadolinium enhancement 2 years after surgery. 


\section{Case Reports in \\ Ophthalmology}

Case Rep Ophthalmol 2019;10:319-326
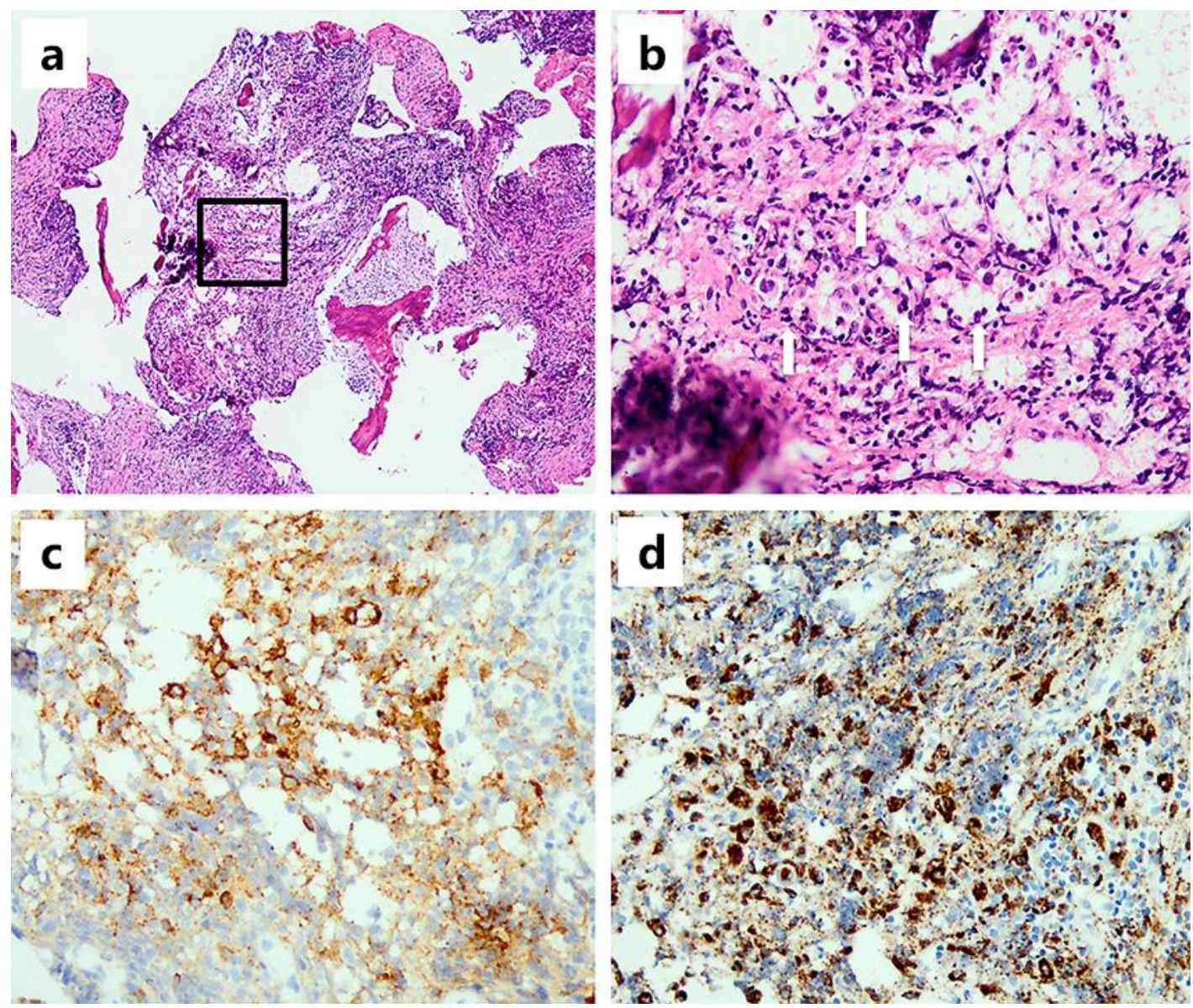

Fig. 3. Histological examinations. a The tumour comprised Langerhans cells and was mixed with non-specific inflammatory cells. Haematoxylin-eosin staining. $\times 100$. Inset This indicates the area shown in image b. b The nuclei of the Langerhans cells displayed a typically cleaved and indented appearance (arrows). Haematoxylin-eosin staining. $\times 400$. $\mathrm{c}$ The tumour cells were positive for CD1a. Immunohistochemistry. $\times 400$. $d$ The tumour cells were positive for S100. Immunohistochemistry. $\times 400$. 